\title{
Kommentare
}

\section{Erwin Fischer \\ Das Bundesverfassungsgericht und das Gebot der Trennung von Staat und Kirche}

Wer sich unbefangen in der Bundesrepublik Deutschland umsieht, käme nicht auf den Gedanken, daß unser Grundgesetz eine grundsätzliche Trennung von Staat und Kirche vorsieht - nur grundsätzlich infolge der beiden im Grundgesetz zugelassenen Ausnahmen: Religionsunterricht in den öffentlichen Schulen und Kirchensteuerprivileg.

Kreuze in vielen öffentlichen Gebäuden, vornehmlich in Gerichten und Schulen, dazu Schulgebete und Schulgottesdienste, zwar nicht überall, aber doch in den meisten Bundesländern. Beamtete Pfarrer in der Bundeswehr und im Strafvollzug. Unentgeltliche Dienstleistungen für die beiden christlichen Großkirchen durch Ausbildung der Pfarrer in theologischen Fakultäten oder Fachbereichen der Universitäten. Treueeid der Bischöfe bei Amtsübernahme.

Peter von Tiling hat seinem Beitrag: "Die Kirche in der pluralistischen Gesellschaft « in der Zeitschrift für evangelisches Kirchenrecht ${ }^{\prime}$ ein Verzeichnis der institutionalisierten Mitwirkungsrechte der Kirchen im staatlichen Bereich als Anhang beigefügt: insgesamt 17 Seiten. Diese bedürften aber noch der Ergänzung. Diese enge Verbindung zwischen Staat und Kirche entspricht dem Übergewicht der sich ausdrücklich »christlich « nennenden Parteien: Sie fordern erneut, daß christ liche Werte nicht nur im privaten, sondern auch im öffentlichen Bereich Anerkennung finden und in Gesetzen berücksichtigt werden müssen. Es darf jedoch nicht übersehen werden, daß auch sozialliberal oder sozialdemokratisch regierte Länder an dieser Mißachtung des Grundgesetzes mitgewirkt haben.

Diese Entwicklung steht in eklatantem Widerspruch zur Stellung der Kirchen in unserer Gesellschaft nach dem Zeugnis von Autoren, die sich im kirchlichen Bereich betätigen oder ihm nahestehen. Bereits 1957 hat Romano Guardini festgestellt, daß "sich vom Beginn der Neuzeit an eine nichtchristliche Kultur herausarbeitet «." Ähnlich hat sich Karl Rahner geäußert, der Deutschland als ein "Heidenland mit christlicher Vergangenheit und christlichen Restbeständen « bezeichnet hat, als ein "Land, das zum Missionsland geworden sei «. ${ }^{3}$ Konrad Hesse, Verfasser kirchengünstiger Gutachten in staatskirchenrechtlichen Verfahren vor dem Bundesverfassungsgericht, sodann von 1975 bis 1987 Bundesverfassungsrichter, schrieb 1965: "Wenn die Kirchen trotz dieser inneren Krise eine Position äußerer Stärke anstreben und gewonnen haben, so ist das in gewisser Weise folgerichtig: sie suchen das, was sie an unmittelbarem Einfluß auf die moderne Gesellschaft verloren haben, mittelbar durch staatskirchenrechtliche Institutionalisierung zurückzugewinnen. «4 
Dies gilt auch heute noch, soweit es sich um die Machtpositionen der beiden christlichen Großkirchen im Gegensatz zur Entchristlichung der Gesellschaft handelt. Das jüngste Beispiel: Die aufgrund des Bayer. Konkordats vom 24. 3. 1924/ 7. 7. 1978 errichtete Kirchliche Gesamthochschule Eichstätt mit "Schwerpunkt Lehrerbildung" erhält die Bezeichnung »Katholische Universität in kirchlicher Trägerschaft« und wird durch die Studiengänge Mathematik, Geographie und Wirtschaftswissenschaften erweitert. Der Staat ersetzt $90 \%$ der tatsächlichen Aufwendungen einschließlich Investitionen (Bay. GVBl. 1988, 24I).

Seit einigen Jahren ist es Mode geworden, von einer religiösen Renaissance zu sprechen. So meint Hans Küng, gegenwärtig sei »ein Prozeß der Wiederentdeckung der Religion « zu verzeichnen.' Soweit dies zutrifft - nach Küng als Folge der soziokulturellen Krise und in Verbindung mit einem geistigen Aufbruch -, spielt sich dieser Prozeß sicherlich nicht im Rahmen der Amtskirchen ab, sondern in Opposition zu ihnen, wenn nicht sogar das Christentum als Grundlage neuer Religiosität überhaupt verlassen wird. Vor allem ist aber zu bedenken, daß das grundgesetzlich gesicherte Trennungsprinzip nicht Ausdruck von Religionsfeindlichkeit ist, da nämlich die rechtliche Grundlage der Trennung von Staat und Kirche die Religionsund Weltanschauungsfreiheit bildet, woraus sich auch die Freiheit der Kirchen im Staat ergibt. ${ }^{6}$

\section{Es besteht keine Staatskirche}

Was sagt nun unser Grundgesetz zum Verhältnis von Staat und Kirche und - um die Kardinalfrage gleich zu stellen - wie werden die verfassungsrechtlichen Bestimmungen vom Bundesverfassungsgericht, nicht zu Unrecht als Hüter der Verfassung apostrophiert, ausgelegt? In den grundlegenden Entscheidungen vom I4. I 2. 1965 ist ausgeführt: "Das Grundgesetz legt durch Art. 4 Abs. I, Art. 3 Abs. 3, Art. 33 Abs. 3 GG sowie durch Art. 136 Abs. I und 4 und Art. 137 Abs. I WRV in Verbindung mit Art. 140 GG dem Staat als Heimstatt aller Staatsbürger ohne Ansehen der Person weltanschaulich-religiöse Neutralität auf. Es verwehrt die Einführung staatskirchlicher Rechtsformen und untersagt auch die Privilegierung bestimmter Bekenntnisse «? Z Zur Begründung ist auf insgesamt sechs verfassungsrechtliche Bestimmungen verwiesen worden, insbesondere auf die unverletzliche Religions- und Weltanschauungsfreiheit, ferner auf das aus der WRV übernommene Gebot: "Es besteht keine Staatskirche« (Art. I 37 Abs. I WRV). Umfassender hätte die Pflicht des Staates zu weltanschaulich-religiöser Neutralität nicht begründet werden können. In den erwähnten Entscheidungen hat das Bundesverfassungsgericht geklärt, daß weder juristische Personen noch in glaubensverschiedenen Ehen der (die) einer kirchensteuerberechtigten Kirche nicht angehörende Ehepartner (-in) zur Zahlung der Kirchensteuer für den kirchentreuen Ehepartner verpflichtet sind, da die Kirchenmitgliedschaft Voraussetzung der Kirchensteuerpflicht ist. In der schriftlichen Begründung ist die sich aus dem Neutralitätsgebot ergebende Trennung von

5 "Südwestpresse « vom 15.6 .1988

6 Henning Zwırner hat in seınem grundlegenden Beıtrag "Zur Entstehung der Selbstbestımmungsgarantıe der Religıonsgesellschaften I. J. I848/49" (Zettschrift der Savıgny-Stiftung für Rechtsgeschıchte, IO4 Bd. S. $210 / 295-1987$ ) nachgewiesen, daß man im deutschen Katholizısmus, solange die Anschauungen des Jahres 1848 - eingegangen in Art. 147 RV 5849 , sodann in Art. 137 Abs. 3 WRV - noch lebendig waren, die enge Verbındung zwischen Religıonsfreihest und der daraus folgenden Trennung von Religıon und Kirche vom Staat anerkannt hat (a.a. O. S. 293 ff.).

7 BVerfGE 19, 206-288. Der Autor dieses Beıtrags hat in dreı Verfahren Beteiligte vertreten. Vgl. zur Grundposition die erste Auflage meınes Buches: Erwın Fischer, „Trennung von Staat und Kirche«, 1964. 
Staat und Kirche nicht erwähnt; sie ist aber in den Entscheidungen vom 28.4. 1965 und 25 . I I. $1980 \mathrm{zu}$ finden. ${ }^{8}$

Drei weitere Aussagen in diesen Entscheidungen sind bedeutungsvoll: I. Die Tradition staatlicher Förderung der Kirchen ist schon ihrer Natur nach nicht geeignet, sich gegenüber einer entgegenstehenden verfassungsrechtlichen Neuordnung durchzusetzen. ${ }^{9}$ 2. Die aufgrund der Verfassung erlassenen landesrechtlichen Bestimmungen über die Erhebung von Kirchensteuern müssen im Einklang mit den Verfassungsrechtssätzen, namentlich den Grundrechten des Grundgesetzes stehen. 3. Der Landesgesetzgeber darf sich nicht über das in diesen Verfassungsnormen zum Ausdruck kommende Wertsystem hinwegsetzen. ${ }^{10}$ Diese Entscheidungen des Bundesverfassungsgerichts wirkten wie ein Fanal: Bestürzung bei den Kirchen, die sogar von einem Privilegienverzicht sprachen, ${ }^{11}$ Genugtuung auf seiten der wenigen Staatsrechtslehrer, die schon stets unter Hinweis auf die intellektuelle Redlichkeit verlangt hatten, zur juristischen Methode im Staatskirchenrecht zurückzukehren. ${ }^{12}$ Obermayer sprach daher 1967 von "Staat und Kirche im Wandel $\ll^{13}$, und Quaritsch meinte, daß »die deutsche Staatskirchenrechtslehre die Anker zu neuer Fahrt gelichtet« habe; er ging von einer zweiten Ära nunmehr grundgesetzlich orientierten Staatskirchenrechts aus. ${ }^{14}$

Im Hinblick auf spätere, davon abweichende Entscheidungen des Bundesverfassungsgerichts ist festzustellen, daß die Aussage über die weltanschaulich-religiöse Neutralität des Staates auch in der weiteren Rechtsprechung beibehalten wurde, zuletzt in der Entscheidung vom 25.02. 1987 über die Teilnahme von Schülern eines anderen Bekenntnisses am Religionsunterricht. ${ }^{15}$

Die Neutralitätsverpflichtung hat aber nicht nur Bedeutung für das Individuum, das sich auf die unverletzliche Religions- und Weltanschauungsfreiheit berufen kann, sondern auch für die Religionsgesellschaften (Kirchen), deren autonome Stellung, die sogenannte Kirchenfreiheit, gleichfalls aus Art. 4 GG abzuleiten ist, sowie für Weltanschauungsgemeinschaften. Zu der primär garantierten Individualsphäre hat das Bundesverfassungsgericht seit 1960 in ständiger Rechtsprechung ausgeführt, daß Art. 4 Abs. I und 2 GG "dem Einzelnen einen Rechtsraum " gewährleistet, »in dem er sich die Lebensform zu geben vermag, die seiner Überzeugung entspricht, mag es sich dabei um ein religiöses Bekenntnis oder eine irreligiöse - religionsfeindliche oder religionsfreie - Weltanschauung handeln. ${ }^{16}$

Da die Grundrechte »Abwehrrechte des Bürgers gegen den Staat « sind und diesen in seiner Macht beschränken ${ }^{17}$, ist es konsequent, daß der staatsfreie Raum stets als freier Rechtsraum bezeichnet wird. Es handelt sich daher nicht um einen Bereich im faktischen (räumlichen) Sinn, sondern um einen rechtlich gesicherten Bezirk, der sich aus »der einzigartigen Stellung dieses klassischen, auch durch die kategorische Formulierung des Art. 4 Abs. I und 2 GG ausgezeichneten Grundrechts « ergibt. $^{18}$

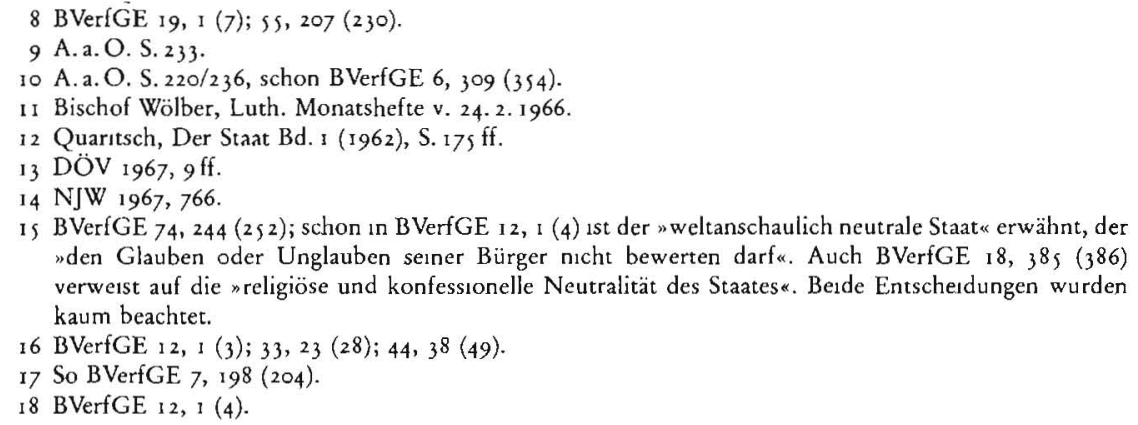


Durch dessen Standort im staatsfreien Rechtsraum ist zugleich die Trennung von Staat und Kirche im Sinne einer Verfahrensgarantie gegeben. ${ }^{19}$

\section{Religionsfreibeit: Trennung von Staat und Kirche}

Der enge Zusammenhang zwischen der unverletzlichen Religions- und Weltanschauungsfreiheit und der Trennung von Staat und Kirche wurde lange Zeit trotz Verpflichtung des Staates zur weltanschaulich-religiösen Neutralität weder vom Bundesverfassungsgericht noch im Schrifttum anerkannt. Bereits 1964 wurde die Argumentation entwickelt, daß sich Freiheit und Selbständigkeit der Kirchen verfassungsrechtlich nur auf Art. 4 Abs. I GG stützen lassen. ${ }^{20}$ Erst vier Jahre später verkündete J. Listl, SJ, Direktor des Instituts für Staatskirchenrecht der Diözesen Deutschlands in einem »Essener Gespräch « als Leitsatz: aus der konsequenten Verwirklichung des in Art. 4 GG gewährleisteten Individual- und Verbandsgrundrechts der Religionsfreiheit folge, daß dieses Grundrecht im Zuge seiner vollen Verwirklichung die aus der WRV übernommenen Garantien (Art. 137, Abs. 1-3) enthalte. Diese Bestimmungen erwiesen sich daher lediglich als deklaratorische Entfaltung der Religionsfreiheit. ${ }^{21}$

Auf dieser Tagung wurde sofort widersprochen, weil aus Art. 4 GG mit der gleichen Begründung auch die Trennung von Staat und Kirche gefordert und extrem säkulare Trennungstendenzen herausgelesen werden können. ${ }^{22}$ Damals lag bereits das im Ergebnis richtige, in der Begründung jedoch verfehlte Schulgebetsurteil des Hessischen Staatsgerichtshofs ${ }^{23}$ vor, in dem das Verbot nicht auf den Trennungsgrundsatz, sondern auf die negative Bekenntnisfreiheit gestützt wurde, der gegenüber der positiven Religionsfreiheit ein Vorrang eingeräumt wurde. Dadurch ist eine für die weitere Entwicklung unheilvolle Aufspaltung des einen Grundrechts - Religionsund Weltanschauungsfreiheit - vorgenommen worden.

\section{Positive und negative Religionsfreibett: Instrument zur Privilegıerung der Kirchen}

Als sich 1967 die deutschen Staatsrechtslehrer mit den "Kirchen unter dem Grundgesetz " befaßten, bildeten die positive und negative Religionsfreiheit einen Schwerpunkt. M. Heckel ${ }^{24}$ vertrat die Auffassung, die Religionsfreiheit werde von gewissen humanistischen, kulturpolitischen und verfassungsrechtlichen Strömungen als Freiheit zur Abkehr von der Kirche und von den staatlichen Staatskircheninstitutionen verstanden, so daß der Sinn der Glaubensfreiheit in der Freiheit, nicht zu glauben, in der Negation oder der sogenannten "negativen « Glaubensfreiheit liege, die man nach dem Maß der Dissidenten und Atheisten zumesse, während für den Gläubigen der großen landeskirchlichen Bekenntnisse - also für die heute noch ganz überwiegende Mehrheit der Bevölkerung - Glauben und Glaubensfreiheit, die "positive» Glaubensfreiheit sozusagen stellvertretend von den großen Institutionen wahrgenommen und auf sie verrechnet werden mit der Konsequenz, daß die Religionsfreiheit auf ein schmales Segment zusammenschrumpfe, sie in "eine Intoleranz der Negation« umschlage. Aus der Freiheit, nicht zum Bekenntnis gezwungen zu

ig H. Goerlich, Grundrechte als Verfahrensgarantien (1981) S. 88, 91, 170, $287 \mathrm{ff}$.

20 "Trennung von Staat und Kirche«, I. A. (1964) S. 40 f. und $167 \mathrm{ff}$.

21 Essener Gespräche, Bd. 3 (1968) S. 135.

22 A. a. O., S. 7 und 139 (unter Hinwers auf Fischer, "Trennung von Staat und Kirche", 1. A. (1964).

23 DÖV 1966, 51; dazu Böckenförde DÖV 1966, S. 30.

24 VVDStRL, H. 26, S. $5,13 \mathrm{ff}$. 
werden, entstehe das $\mathrm{Z}$ wangsrecht, anderen Bekenntnisausübung zu verbieten. Aus der Freiheit der Glaubenslosen in der Erziehung folge, daß auch die anderen glaubenslos erzogen werden müßten. Heckel fand überwiegend Zustimmung ${ }^{2 s}$, obwohl deutlich zu erkennen war, daß die Adjektiva "positiv« und "negativ « auch das Verhalten der Gläubigen einerseits und der Glaubenslosen anderseits bewerteten: Der Glaubensnegierer hindert die gläubige Mehrheit am positiven Glaubensbekenntnis. Daraus ergibt sich die Bedeutung der Kontroverse für das öffentliche Schulwesen, mit dem das Bundesverfassungsgericht nach dem Verzicht auf die öffentliche Bekenntnisschule ab I 967/68 befaßt war. Umstritten waren der christliche Charakter der öffentlichen Grund- und Hauptschulen in Baden-Württemberg (Art. Is I LV) sowie die öffentlichen Volksschulen in Bayern, in denen die Schüler nach den Grundsätzen der christlichen Bekenntnisse erzogen werden (Art. 135 LV), und das Schulgebet. ${ }^{26}$

Sämtliche Verfassungsbeschwerden wurden zurückgewiesen. Die vom Bundesverfassungsgericht erstmals akzeptierte Unterscheidung zwischen negativer und positiver Religionsfreiheit findet man bereits im Leitsatz 3 des zur Landesverfassung Baden-Württemberg erlassenen Urteils, wonach Art. 7 GG es dem demokratischen Landesgesetzgeber überlasse, den religiös-weltanschaulichen Charakter der öffentlichen Schulen, für die religiöse und weltanschauliche Vorstellungen von jeher relevant waren, unter Berücksichtigung von Art. ${ }_{4} \mathrm{GG}$ zu bestimmen sowie das im Schulwesen unvermeidliche Spannungsverhältnis zwischen »negativer « und "positiver « Religionsfreiheit nach dem Prinzip der Konkordanz zwischen den verschiedenen verfassungsrechtlich geschützten Rechtsgütern zu lösen. ${ }^{27}$ Eine weitere Differenzierung rettete die christliche Grund- und Hauptschule im Sinne der badischen Simultanschule mit christlichem Charakter. Zwar dürfe die Schule außerhalb des Religionsunterrichts »nicht christlich-konfessionell fixiert sein «, aber die zulässige „Bejahung des Christentums in den profanen Fächern beziehe sich in erster Linie auf die Anerkennung des prägenden Kultur- und Bildungsfaktors, wie er sich in der abendländischen Geschichte herausgebildet" habe, nicht auf »die Glaubenswahrheit«.

Den eindeutig verfassungswidrigen Schulartikel in der Verfassung des Freistaates Bayern zu rechtfertigen, war schwieriger. Man half mit einer verfassungskonformen Auslegung, obwohl diese nur zulässig ist, wenn "zwei verschiedene Deutungen einer Norm möglich « sind..$^{28}$

In der Schulgebetsentscheidung schlägt das Bundesverfassungsgericht den gleichen Weg ein wie der Hessische Staatsgerichtshof und erklärt, daß »die im Grundgesetz niedergelegten institutionellen Grundsätze des Staats-Kirchen-Verhältnisses keinen primären Maßstab für die Beantwortung der Frage nach der Zulassung des Schulgebets abgeben ${ }^{29}{ }^{29}$ Auch spielt in beiden Entscheidungen die positive und negative Bekenntnisfreiheit eine ausschlaggebende Rolle mit dem einzigen Unterschied: Der Hess. Staatsgerichtshof hat der negativen Bekenntnisfreiheit den Vorrang eingeräumt und daher das Schulgebet für unzulässig erklärt, während das Bundesverfas-

2s Hollerbach, VVDStRL, H. 26, S. 98 ; Scheuner, VVDStRL, H. 26, S. 125 u. a.

26 Die ım Auftrage der Humanistıschen Unıon von betroffenen Eltern erhobenen Verfassungsbeschwerden wurden von mir vertreten. Die Entscheidungen in den Schulsachen ergingen am 17. 12.1978 (BVerfGF $4^{1}, 29$ und 65 zugleich für Nordrhem-Westfalen in gegenläufiger Zielsetzung 41, 89), in der Schulgebetssache am 16. 10. 79 (BVerfGE 52, 223 ).

27 BVerfGE 41,49

28 BVerfGE $35,263 / 280$ sowı bereıts E $8,71 / 78$. Die gesetzeskräftıge Veröffentlichung ım BGBl 1976 I, S. 1040 gem. $\ 31$ Abs 2 BVerfGG wurde be1 der Neufassung des Gesetzes über das Erziehungs- und Unterrichtswesen vom 29. 2. 1988 (GVBl. 1988, S.6I - Art.6) ignoriert.

29 BVerfGE $\{2,223 / 237$. 
sungsgericht umgekehrt der positiven Bekenntnisfreiheit den Vorrang zubilligte und daher das Schulgebet - obwohl ausdrücklich als »religiöser Bekenntnisakt« und als "schulische Veranstaltung " bewertet - als zulässig erachtet, weil in Berücksichtigung religiöser Bezüge in Gemeinschaftsschulen im Spannungsverhältnis zwischen negativer und positiver Bekenntnisfreiheit einem "mißverstandenem Recht auf Schweigen« wegen der gegebenen Ausweichmöglichkeit dank freiwilliger Teilnahme für Schüler und Lehrer auch in Beachtung des Toleranzgebots kein Vorrang zukomme.

\section{Religionsfreibett und Schule}

Alle Grundrechte des status negativus entfalten sich in einem von staatlicher Einflußnahme freien Rechtsraum. Damit ist eine staatfreie Sphäre des Bürgers gegen Eingriffe der öffentlichen Gewalt geschaffen. Der Bürger kann sich insoweit eine Lebensform geben, die seiner religiösen und weltanschaulichen Überzeugung entspricht. ${ }^{3 \circ}$ Dies besagt, daß alle Entscheidungen auf dem Gebiet von Religion und Weltanschauung - "gleichgültig ob es sich um ein religiöses Bekenntnis oder eine religionsfremde oder eine religionsfreie Weltanschauung handelt« - Ausdruck der Freiheit sind, »zu glauben oder nicht zu glauben, d. h. einen Glauben zu bekennen, zu verschweigen, sich von dem bisherigen Glauben loszusagen und einem anderen Glauben zuzuwenden . $^{31}$ Alle Äußerungen sind positiver Ausdruck der einen Religions- und Weltanschauungsfreiheit ohne die Möglichkeit, zu unterscheiden zwischen einem positiven und einem negativen Freiheitsrecht. Soviel zur BegriffskJärung.

Da die öffentliche Schule als Pflichtschule »Staat« ist, darf sich die Religions- und Weltanschauungsfreiheit in der öffentlichen Schule überhaupt nicht entfalten. Die Schule ist daher auch nicht der Ort, wo, um einmal die verfehlten Begriffe zu gebrauchen, positive und negative Bekenntnisfreiheit aufeinanderstoßen könnten. Ein Spannungsverhältnis ist nämlich nicht gegeben und somit auch nicht ausgleichsbedürftig.

Ein »Freiraum« für die Ausübung der »positiven Bekenntnisfreiheit« in der öffentlichen Schule, von dem das Bundesverfassungsgericht spricht ${ }^{32}$, ist daher undenkbar. Wenn es kurz zuvor ausführt, der Staat gebe »hier der positiven Bekenntnisfreiheit Raum in einem Bereich, den er ganz in seine Vorsorge genommen hat und in welchem religiöse und weltanschauliche Vorstellungen von jeher relevant waren «, so widerspricht dies sowohl dem durch die Bekenntnisfreiheit geschaffenen Freiraum als auch der Verpflichtung des Staates zu Neutralität im Bereich von Religion und Weltanschauung. Denn der Freiraum ist der Privatsphäre, aber auch dem gesellschaftlichen und kirchlichen Bereich zugeordnet, während religiöse und weltanschauliche Vorstellungen im staatlichen Bereich - hier Schule - nichts zu suchen haben.

Auch aus dem wiederholten Hinweis auf Art. 7 Abs. 3 GG sind nicht die richtigen Schlüsse gezogen worden. Bei dem Religionsunterricht handelt es sich um eine Ausnahmevorschrift, um einen systemwidrigen Fremdkörper, nach Auffassung von Mahrenholz ${ }^{33}$ um ein "Fossil alter Zeiten in der Nähe von Staat und Kirche«. Sein Ausnahmecharakter und zugleich seine Zulässigkeit ergeben sich aus drei Bestim-

30 S. Fn. I6.

31 BVerfGE 24, 236, 245

32 BVerfGE $\{2,223 / 24$.

33 Die Kirche in der Gesellschaft der Bundesrepublik, 2. A. (1972) S. 182. 
mungen: Sein Inhalt wird von den Religionsgemeinschaften festgelegt; für die Schüler ist er Wahlfach; für die Lehrer besteht kein Lehrzwang. Daraus ergibt sich der zwingende Schluß, daß auch in der öffentlichen Schule die unverletzliche Religions- und Weltanschauungsfreiheit zu beachten und eine Berufung auf die Tradition nicht möglich ist.

Gerade die Regelung des Religionsunterrichts in der öffentlichen Schule zeigt, daß die sich aus dem Neutralitätsgebot und der Religions- und Weltanschauungsfreiheit ergebende Trennung von Staat und Kirche weder als eingeschränkt noch als abgeschwächt betrachtet werden kann, sondern als eine grundsätzliche Trennung mit wenigen Ausnahmen, die daher eng auszulegen sind und vor allem keine Schlüsse auf eine irgendwie geartete Partnerschaft von Staat und Kirche erlauben.

\section{Partnerschaft von Kirche und Staat?}

Partnerschaft ist auch das Stichwort für eine weitere kritische Stellungnahme zu Ausführungen in der Begründung des Urteils vom 21.09. 1976, soweit sie sich auf das »in Art. ${ }_{140}$ GG niedergelegte Verhältnis zwischen Staat und Kirche» beziehen. ${ }^{34}$ Zunächst ist zu beanstanden, daß als Rechtsquelle für dieses Verhältnis lediglich Art. 140 GG angegeben ist, jedoch nicht Art. 4 Abs. I GG, der im Urteil vom I4. 12. I965 die Grundlage für die Verpflichtung des Staates zu weltanschaulich-religiöser Neutralität bildet. ${ }^{35}$ Denn Art. ${ }_{4}$ GG ist auch die Rechtsgrundlage für die Freiheit der Kirche, auf die sich das Gericht in seiner Entscheidung bezogen hat. ${ }^{36}$ Aber aus der Kirchenfreiheit läßt sich die in der Urteilsbegründung erwähnte Koordinationslehre oder Partnerschaft nicht ableiten. Daher ist zum Verständnis einer in die Vergangenheit führenden Entwicklung kurz das Koordinationssystem seit seiner Entstehung zu skizzieren. Es hängt eng mit dem Begriff »societas perfecta« zusammen. Bereits im 5. Jahrhundert entstand die Vorstellung der $»$ societates perfectae der Kirche und des Staates « und zugleich »die Lehre von der Superiorität des geistlichen über das weltliche Recht «. ${ }^{37}$ Diese Koordinationslehre wurde von Papst LeoXIII. in verschiedenen Lehrschreiben ( $885 / 90)^{38}$ bestätigt, indem »die Kirche ebenso wie der Staat selbst « als »eine nach Wesen und Recht vollkommene Gesellschaft« bezeichnet werden.

H. Peters ${ }^{39}$ hat sich als Korreferent auf der Staatsrechtslehrertagung 1952 dieser Auffassung angeschlossen und im Zusammenhang damit den "Bürger zweier Welten « erwähnt, allerdings zunächst mit Beschränkung auf den »kirchlich-g)äubigen Staatsangehörigen «. K. Hesse ${ }^{40}$ ging noch weiter und sprach 1956 von der »nunmehrigen Anerkennung der gleichberechtigten Partnerschaft der Kirchen durch den Staat« und deren "gemeinsame Verantwortlichkeit für die öffentliche Ordnung « auf der Grundlage, daß »die Menschen, die sie zu einem Gemeinwesen verbinden, dieselben sind «. Sodann verkündete Mikat ${ }^{41}$ I 960 als das »heutige kirchenpolitische System " die Koordinationslehre, wonach Kirche und Staat als gleichrangige Gemeinschaften erster Ordnung, als »societates perfectae" zum Gemeinwohl zusammenwirken, eine Lehre, die der Bundesgerichtshof in seine Entscheidung vom

\footnotetext{
34 BVerfGE 43, 3 I 2 betrifft Inkompatibilität zwıschen gesstlichem Amt und Abgeordnetenmandat. 35 BVerfGE 19, 206 (216).

36 BVerfGE 42, 3 I 2 (345).

37 J. Klein, Grundlagen und Grenzen des kanonischen Rechts (1947) S.9.

38 H. Beckel, Christliche Staatslehre. Dokumente (r96I) S. i 3 ff., S. $32 \mathrm{ff}$.

39 VVDStRL H. 11 , S. 178.

40 Der Rechisschutz durch staatliche Gerıchte im kirchlichen Bereıch (Göttıngen 1956) S. 72.

$4^{\text {I }}$ Bettermann-Nipperdey-Scheuner, Die Grundrechte Bd. IV, 1. Halbbd. (1960) S. 11 I ff. (S. 145).
} 
I. 3. 196I wie folgt übernahm: "Das Grundgesetz geht von der grundsätzlichen Gleichordnung von Staat und Kirche als eigenständigen Gewalten aus. Die Kirchen sind der staatlichen Hoheitsgewalt nicht mehr unterworfen und regeln ihre Angelegenheiten selbständig und in eigener Verantwortung. « $^{42}$

Auch im Hinblick auf die eindeutige Absage an die Tradition der Verbindung von Staat und Kirche durch das Bundesverfassungsgericht ${ }^{43}$ ist erstaunlich, daß im Bremer Mandatsurteil zum Verständnis des in Art. I 40 GG niedergelegten Verhältnisses von Staat und Kirche die geschichtliche Kontinuität herangezogen und »die besondere Bedeutung der Kirchen für das Leben in Staat und Gesellschaft anerkannt wird." Daraus entstehe für Staat und Kirche, "die sich für dieselben Menschen verantwortlich fühlen«, die "Notwendigkeit verständiger Kooperation «. ${ }^{44} \mathrm{Zur} \mathrm{Be}-$ gründung werden nun nicht etwa Rechtsprechung und staatskirchenrechtliches Schrifttum herangezogen, sondern Regierungserklärungen der sozialdemokratischen Bundeskanzler Brandt und Schmidt (1973/1974), die den Wunsch nach Partnerschaft mit den Kirchen »in ihrer notwendigen geistigen Wirkung « enthalten. Das Bundesverfassungsgericht fügte hinzu: »Im Grund ist das auch gemeint, wenn das Grundverhältnis zwischen Staat und Kirche in der Bundesrepublik Deutschland als Verhältnis einer hinkenden Trennung،, als wechselseitige Selbständigkeit innerhalb eines Koordinationssystems oder als Partnerschaft zwischen Kirche und Staat charakterisiert wird. "In der weiteren Begründung wird in der "Schrankenformel des Art. ${ }_{37}$ Abs. 3 WRV heute die Basis für eine Konkordanz zwischen staatlicher und kirchlicher Ordnung erblickt«, so daß »staatliche Gesetze nicht die den Kirchen wesentlichen eigenen Ordnungen beeinträchtigen und daß kirchliche Gesetze nicht die für den Staat unabdingbare Ordnung kränken werden. $\star^{45}$

Diese Ausführungen befinden sich im Einklang mit der bereits zitierten Verantwortung von "Staat und Kirche für dieselben Menschen und dem Bekenntnis zum Koordinationssystem und einer Partnerschaft zwischen Kirche und Staat «. Dies entspricht in etwa der überkommenen katholischen Koordinationslehre, die von Mikat aufgewärmt wurde und vielen Autoren mit dem Slogan »idem civis et christianus« als Argument dient. ${ }^{46}$

1984 greift Kardinal Ratzinger ${ }^{47}$ in einem umfangreichen Beitrag auf die älteste Version der Koordinationslehre zurück. Er bezeichnet den modernen Staat zweimal als "societas imperfecta" und bietet, aufgrund der Superiorität der Kirche, dem unvollkommenen Staat "Kräfte von außerhalb seiner selbst« an, »um als er selbst bestehen zu können«. Kardinal Ratzinger hielt die Zeit für einen Appell gekommen, zur Koordination von Staat und Kirche zurückzukehren und in Ignorierung, ja Negierung der Verpflichtung des Staates zu weltanschaulich-religiöser Neutralität seine Unvollkommenheit durch Zusammenarbeit mit der Kirche auszugleichen. Dies hängt sicherlich damit zusammen, daß das Bundesverfassungsgericht nicht nur in dem Bremer Mandatsurteil, sondern in weiteren Entscheidungen der letzten Jahre der Kirche eine Stellung eingeräumt hat, die sie als eine neben dem Staat stehende Organisation anerkennt.

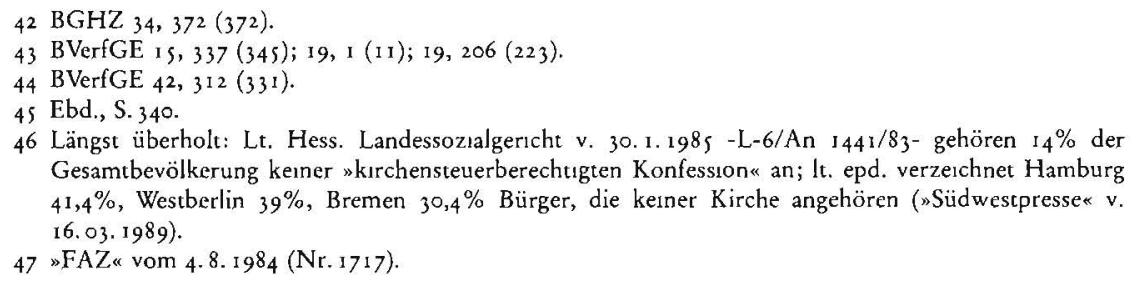


Dies ergibt sich bereits aus den Begriffen Koordination und Partnerschaft, die einen gleichen Rang mit dem Staat voraussetzen. Außerdem ist der Begriff Koordinationslehre seit jeher mit der Vorstellung verbunden, daß es sich bei Staat und Kirche um voneinander unabhängige, souveräne und kommensurable Organisationen handelt. Davon kann jedoch keine Rede sein; denn die Unterordnung der Kirchen unter die Hoheit des Staates ist prinzipiell erhalten geblieben..$^{8}$

\section{Kirchenfreibeit - keine zusätzlichen Rechte}

Den kirchenfreundlichen Entscheidungen des Bundesverfassungsgerichts liegt der bereits erwähnte Begriff Kirchenfreiheit zugrunde. Er ist aus Art. 4 Abs. 1 und 2 GG abzuleiten. ${ }^{49}$ Diese Grundgesetzbestimmungen garantieren zunächst ein individuelles, subjek tives und höchstpersönliches Grundrecht, das »dem Staat den bestimmenden Zugriff auf die religiöse oder weltanschauliche Dimension des Menschen verwehrt. ${ }^{\text {so }}$ Nun unterscheidet sich aber die kooperative Garantie der Kirchenfreiheit von der Religions- und Weltanschauungsfreiheit als Individualrecht in verschiedener Hinsicht. Vor allem ist festzustellen, daß der individuellen Religions- oder Weltanschauungsfreiheit insofern Vorrang zukommt, als sie unverletzlich ist und im Gegensatz zur Kirchenfreiheit nicht durch das für alle geltende Gesetz eingeschränkt ist. Die schwierige Beantwortung der Frage, welches Gesetz für jedermann verbindlich ist und welches als zur kircheneigenen Sphäre gehörend keiner Beschränkung unterliegt, ergibt sich aus der Auslegung des Begriffs »ungestörte Religionsausübung “. Da Art. I 37 Abs. 3 WRV sich »als deklaratorische Entfaltung des Art. 4 Abs. I und 2 GG erweist « (Listl), folgt zwingend, daß unter Berufung auf die Kirchenfreiheit in keinem Fall mehr an Freiheit beansprucht werden kann als aus der individuellen Religionsfreiheit abzuleiten wäre. Daraus ergibt sich, daß der Raum, in dem das für alle geltende Gesetz nicht gilt, jener unserem Staat fremde Bereich ist, "der nach Inhalt und Wesen vom Bekenntnis durchwaltet ist und seine Gestalt durch den Vollzug des überirdischen Auftrags, also Dogma und Kultus, Verfassung sowie Rechte und Pflichten der Mitglieder betrifft "st

In dieser Sphäre gelten allein die Entscheidungen der Religionsgesellschaften. Hieraus folgt aber, daß eine Angelegenheit der Religionsgesellschaften ihren kirchlichen Eigencharakter verliert, »wenn sie aus dem innerkirchlichen (sinternen $)$ Bereich in die Welt des Staates und des Staatsbürgers hineinwirkt«. Weitere Folge ist, $\mathrm{da}$, »was in diesem Raum geschieht, nicht dem partikulären Selbstverständnis der Religionsgesellschaft überlassen werden« kann, weil "sonst dem einzelnen der Schutz der Verfassung versagt bliebe, die in dieser Sphäre die einzige, jede andere ausschließende Lebensordnung bildet «. ${ }^{52}$

Nun hat das Bundesverfassungsgericht in späteren Entscheidungen den den Kirchen uneinschränkbaren Bereich soweit erstreckt, daß »maßgebendes Kriterium für die Zuordnung einer Einrichtung zur Kirche danach nicht etwa die Zugehörigkeit zur Kirchenverwaltung « ist; »es genügt vielmehr, daß die in Frage stehende Einrichtung der Kirche so nahe steht, daß sie teilhat an der Verwirklichung eines Stücks Auftrag der Kirche im Geist christlicher Religiosität, im Einklang mit dem Bekenntnis der christlichen Kirche und der Verbindung mit den Amtsträgern der Kirche (vgl.

48 Quaritsch, Der Staat, Bd. I (1962) S. 292. Seıne Bezugnahme auf Hesse in Fn. 83 ist jedoch verfehlt, da dieser sıch gegen eine Unterordnung ausgesprochen hat (s. Rechtsschutz S. 72 ff.).

49 Listl in *Essener Gespräche» 3 (1968), S. 45.

so BVerfGE 42, $312(322)$.

5I Quaritsch, Der Staat, Bd. I (1962) S. 295.

52 BVerfGE 53, 366 (392): mißbilligt staatliche Organısatıon konfessioneller Krankenhäuser. 
BVerfGE ${ }_{46}$, 73/87). Die so gewährleistete Freiheit der Kirche im Staat schließt ein, daß sich die Kirche zur Erfüllung ihres Auftrags auch der Organisationsformen des staatlichen Rechts bedienen kann, ohne daß dadurch die Zugehörigkeit der auf dieser Rechtsgrundlage gegründeten Einrichtung zur Kirche aufgehoben würde«. sz

Wie aus der Begründung eindeutig hervorgeht, legt das Bundesverfassungsgericht seinen Ausführungen nicht nur mit Recht Art. 4 Abs. 2 GG zugrunde, sondern es bezieht sie ausschließlich auf die beiden Großkirchen. Völlig ignoriert wird, daß die so gewährte Freiheit der Kirche sämtlichen Religionsgesellschaften und Weltanschauungsvereinigungen zusteht. Eine Vielfalt möglicher Eigenverständnisse für die Verwirklichung der durch Art. 4 Abs. 2 GG geschützten Religionsausübung ist daher denkbar, auch im Hinblick auf die Zunahme eines religiösen Fundamentalismus, der nicht nur im Iran beheimatet ist. Denn jedenfalls sind Verfassungsbegriffe "für alle Bekenntnisse und Weltanschauungen gleich zu interpretieren «. 33 Schon diese unumgängliche Ausweitung verbietet es, unter Verzicht auf die Souveränität des Staates, den Umfang der Religionsausübung dem sogenannten Selbstverständnis der Religionsgesellschaften und Weltanschauungsvereinigungen zu überlassen.

Auch wenn davon abgesehen wird, daß konfessionelle Krankenhäuser sich verschiedener Organisationsformen bedienen ${ }^{54}$, ist ein wesentlicher Unterschied zum Sachverhalt im sogenannten Lumpensammlerfallss festzustellen: Die Mitglieder der Vereinigung katholischer ländlicher Jugend haben bei ihren Aktionen im Geist katholischer Religiosität gehandelt und so "ein Stück Auftrag der Kirche in dieser Welt « wahrgenommen. In einem konfessionellen Krankenhaus kann man aber davon ausgehen, daß dies auf die Caritas der meist noch geringen Zahl der Ordensschwestern zutrifft. Diese stellen aber nur weine Minderheit des Personals $\mathrm{dar}$, das weit überwiegend aus Arbeitnehmern besteht, die in gleicher Weise angestellt, beschäftigt und entlohnt werden wie in anderen Krankenhäusern auch. Dies gilt auch für die Ärzteschaft « ${ }^{56}$

Die Frage stellt sich daher, was soll durch Art. 4 Abs. I und 2 GG geschützt werden? In Betracht kommt doch nicht nur die kleine Minderheit der Ordensschwestern, sondern die weit überwiegende Mehrheit der Mitarbeiter, für die ihre Tätigkeit in einem konfessionellen Krankenhaus nicht karitativ bestimmt ist und die sich nicht bis in ihre Privatsphäre hinein einer kirchlichen Dienstgemeinschaft verpflichtet fühlen.

\section{Konfessionelle Krankenhäuser - ım Dienst von Religıonsausübung?}

Richter Rottmann weist darauf hin, daß die konfessionellen Krankenhäuser bei Verzicht auf staatliche Förderung sich staatlicher Struktur entziehen können. Fest steht jedoch, daß zur wirtschaftlichen Sicherung der konfessionellen Krankenhäuser staatliche Förderung nötig ist. Dadurch wird aber der »kirchliche Freiraum « nicht »unterlaufen «, wie Scheuner in den Essener Gesprächen über die karitative Tätigkeit der Kirchen im Sozialstaat ausgeführt hat. ${ }^{57}$ Nun bildet aber Art. 4 GG die verfassungsrechtliche Grundlage für einen kirchlichen Freiraum. Wenn daher ein konfessionelles Krankenhaus als Religionsausübungsbetrieb zu gelten hätte, wäre eine

\$3 BVerfGE I 2, \$4 (\$4).

\$4 Zur Lage in Nordrheın-Westfalen Diözesancarıtasdirektor Kessels ın: „Essener Gespräche« 8 (1973) S. 128.

55 BVerfGE 24, 236 (235 ff.): karıtatıve Tätıgkeıt fällt unter Art. 4 Abs. 2 GG.

56 Zitat aus der abweichenden Meinung des Richters Dr. Rottmann zu der Entscherdung BVerfGE 53, 366 (408/419).

57 "Essener Gespräche" 8 (1973) S. 61 
staatliche Förderung unzulässig. Es ist daher widersprüchlich, einerseits die staatliche Förderung zu billigen, andererseits das konfessionelle Krankenhaus als einen Bereich zu behandeln, der "in der durch Art. 4 Abs.2 GG als unverletzlich gewährleisteten Glaubens- und Bekenntnisfreiheit wurzelt und sich in der durch Art. 4 Abs. 2 GG geschützten Religionsausübung verwirklicht «. ${ }^{8}$

Dies wäre verfassungswidrig. Denn das aufgrund des Sozialstaatsprinzips verfolgte Ziel, eine optimale Krankenhausversorgung der Bevölkerung zu bieten, rechtfertigt die staatliche Unterstützung nur, wenn der konfessionelle Krankenhausbetrieb nicht als Unternehmen im Dienste der Religionsausübung betrachtet und behandelt wird. Auch aus der Sicht des Patienten handelt es sich um kein Unternehmen im Dienste der Religionsausübung, da auch das konfessionelle Krankenhaus sich »jede Handreichung von den Privatpatienten, den Krankenkassen oder den Steucrzahlern entgelten « läßt ${ }^{s 9}$, abgesehen davon, daß »der kranke Bürger angesichts der kostenbedingten Knappheit an Krankenhausbetten auch auf Leistungen konfessioneller Krankenhäuser angewiesen ist, auch wenn er religiösen Implikationen seiner Krankenversorgung gleichgültig oder gar ablehnend gegenübersteht $\ll{ }^{60}$ Entsprechend gilt dies auch für das in diesen Krankenhäusern beschäftigte Personal.

Nicht zuletzt widerspricht es der Struktur eines zeitgemäßen Krankenhauses, es als Anstalt zur kooperativen Ausübung der Religion zu betrachten und somit als einen von staatlicher Einflußnahme freien Rechtsraum von der Anwendung des für jedermann geltenden Gesetzes freizustellen. Vor allem darf nicht ignoriert werden: »Glaubens- und Bekenntnisfreiheit sind nämlich angelegt auf die Gemeinschaft der Gläubigen als eine Verwirklichung in einer sichtbaren Religionsgesellschaft « ${ }^{61}$ Von einer solchen kann jedoch bei einem konfessionellen Krankenhaus in keiner Weise gesprochen werden. Dazu reicht auch das wiederholt von den Entscheidungen des Bundesverfassungsgerichts berufene "Stück Auftrags der Kirche in der Welt ${ }^{62}$ nicht aus.

Seit 1976 erscheint in der Bundesverfassungsgerichtsrechtsprechung der Begriff Kirchenfreiheit bzw. Freiheit der Kirchen ${ }^{6}$, obwohl im Schriftum dieser Begriff seit 1964 im Hinblick auf die Auslegung von Art. I 37 Abs. 3 WRV gebraucht wird. ${ }^{64}$ Dies hängt damit zusammen, daß in der ersten Entscheidung mit Erwähnung der Kirchenfreiheit ${ }^{6 s}$ diese lediglich als »Teilaspekt« (Verbindung von Art. I40 GG mit Art. ${ }_{4}$ GG) betrachtet wurde, während für das Grundverhältnis zwischen Staat und Kirche die Verbindung von Art. I40 GG mit Art. I 36, I37, I 38, 139 und I4 I WRV als entscheidend erschien, und zwar ohne Bezugnahme auf Art. 4 GG.

Auch bildet in dieser Entscheidung die Vorstellung einer wechselseitigen Selbständigkeit von Staat und Kirche im Sinne der Koordinationslehre einen wesentlichen Entscheidungsfaktor, wie sich aus der »Freiheit der Kirchen in ihrer historisch gewordenen Gestalt zum Bekenntnis gemäß ihrem Auftrag« ergebe. Gerade umgekehrt ist nunmehr durch Verbindung zwischen Art. 137 Abs. 3 WRV als deklatorische Entfaltung von Art. 4 Abs. I und 2 GG eine verfassungsrechtliche Grundlage für die Kirchenfreiheit geschaffen worden, der gegenüber die Tradition der Koordinationslehre völlig bedeutungslos geworden ist.

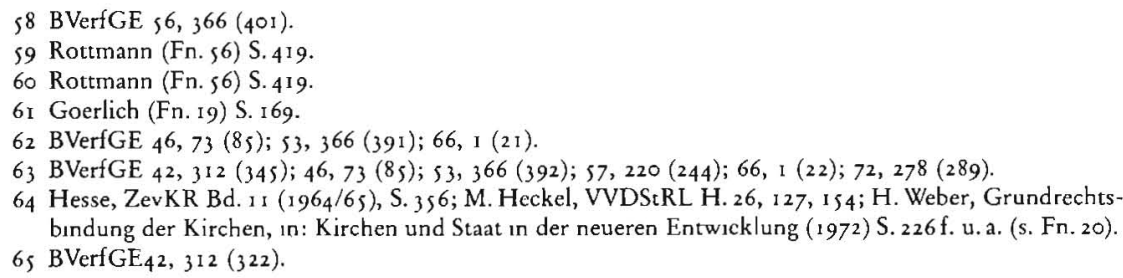


Aus der weiteren Rechtsprechung zur Auslegung der Kirchenfreiheit - meist in Anwendung auf konfessionelle Krankenhäuser - geht hervor, daß es eines Rückgriffs auf die Koordinationslehre gar nicht bedarf, um den beiden christlichen Großkirchen auf einem für den Sozialstaat wichtigem Gebiet eine Machtposition zuzubilligen, die sich sogar auf ein Grundrecht zu stützen vermag. Denn nach dem sogenannten Selbstverständnis der Kirchen fielen nicht nur sämtliche konfessionellen Krankenhäuser, sondern auch konfessionelle Kindergärten, Sozialstationen und Altenheime unter Art. 4 Abs. 2 GG als Stätten der Religionsausübung.

Seit der Entscheidung vom 25.03.198066 betrachtet das Bundesverfassungsgericht die allgemein anerkannte und legitime karitative Betätigung als Religionsausübung, geschützt durch Art. 4 Abs. 2 GG. Diese Feststellung dient mittels des Begriffs Kirchenfreiheit der Auslegung von Art. 137 Abs. 3 WRV mit dem Hinweis, daß die Kirchen aufgrund ihres Selbstverständnisses souverän bestimmen, welcher Bereich von der Kirchenfreiheit erfaßt werde. Dadurch werden die "Schranken des für alle geltenden Gesetzes « weitgehend bedeutungslos, weil Religion in einem dem Staat verschlossenen Gebiet ausgeübt wird. Wie aber die Zulässigkeit von Prozessionen und Wallfahrten beweist, ist der öffentliche Bereich mit dem staatlichen Bereich nicht identisch.

Diese verfassungsrechtliche Argumentation müßte aber konsequent durchgeführt werden. Man käme zu dem Ergebnis, daß von der behaupteten Religionsausübung nicht nur die Mitglieder der eigenen Kirche erfaßt würden, sondern auch Andersgläubige und Konfessionslose, die gezwungen sind, auch konfessionelle Krankenhäuser aufzusuchen, um dank ärztlicher Leistung ohne Rücksicht auf den konfessionellen Charakter des Krankenhauses gesund zu werden. Tatsächlich merken die Patienten aber von einer Religionsausübung nicht das geringste. Denn das in jeder, auch in personeller Hinsicht religiös ausgerichtete und sich selbst finanzierende Krankenhaus gibt es nicht. Alle mit den Kirchen irgendwie verbundenen Einrichtungen können bei natürlicher Betrachtung nicht als religiöse, $d$.h. den staatlichen Gesetzen nicht unterworfene Bereiche betrachtet und behandelt werden. Eine Identität von Religionsausübung und konfessionellem Krankenhaus zu behaupten dies wäre die logische Konsequenz einer verfehlten verfassungsrechtlichen Beweisführung -, widerspricht den eindeutigen Fakten.

Wenn die Kirchen sozialpolitische Aufgaben übernehmen und insoweit auf der Verwaltungsebene mit dem Staat zusammenarbeiten, teilweise sogar aufgrund gesetzlicher Vorschriften durch das Vorrangsprinzip privilegiert sind, ist festzustellen, daß dieses Prinzip weitgehend ausgehöhlt ist, weil die Kirchen die subsidiär geschaffenen Einrichtungen nur zu einem kleinen Teil selbst finanzieren und der von Rottmann vorgeschlagene Verzicht auf staatliche Förderung nicht realisiert werden kann.

\section{Ausblick: Kircbliches Arbettsrecht}

Seit der Entscheidung vom 25.3.198067 sind weitere Entscheidungen verkündet worden, denen die Kirchenfreiheit zugrunde liegt. ${ }^{68}$ Im Hinblick auf die bisherigen Ausführungen erübrigt sich eine besondere Stellungnahme. Sie ist auch nicht möglich, weil das wegen der großen Zahl von Beschäftigten bedeutsame kirchliche

66 BVerfGE 53, 366. Die Bundesverfassungsgerıchtsentscheidung BVerfGE 24, 236 betrifft ennen Sonderfall ohne Bezugnahme auf die Kirchenfreibert.

67 S. Fn. 6I.

68 BVerfGE 57,$220 ; 66,1 ; 70,138 ; 72,278$. 
Arbeitsrecht einer gesonderten Erörterung bedürfte. Lediglich zu dem Beschluß vom 4.6. $1985^{69}$, durch den zwei Urteile des Bundesarbeitsgerichts unter Zurückweisung aufgehoben wurden (übrigens mit I bzw. 2 Gegenstimmen), ist zu bemerken, daß nach Auffassung des Gerichts die zur Entscheidung über die Berechtigung einer fristlosen Kündigung berufenen Arbeitsgerichte gehalten sind, die verbindliche Bestimmung, "was >spezifisch kirchliche Aufgaben`sind, was >Nähe< zu ihnen bedeutet, welches die >wesentlichen Grundsätze der Glaubens- und Sittenlehres sind und was als - gegebenfalls schwerer Verstoß - gegen diese anzusehen ist«, zu beachten, insbesondere auch »die vom kirchlichen Selbstverständnis her gebotene Verpflichtung der kirchlichen Arbeitnehmer auf grundlegende Maximen kirchlichen Lebens«. Dadurch unterstünde sogar die private Lebensführung kirchlicher Kontrolle und Einflußnahme. So wären durch die kraft des kirchlichen Selbstverständnisses getroffenen Vorentscheidungen den Arbeitsgerichten die Hände weitgehend gebunden.

Gerade in Hinsicht auf die wiederholt in den Entscheidungen getroffene Feststellung, daß gegen die Verfassung die Kirchenfreiheit verletzt worden sei, ist zu betonen, daß umgekehrt die Arbeitnehmer sich auf die ihnen nach dem Grundgesetz zustehenden Grundrechte berufen können, in erster Linie auf das unverletzliche Grundrecht eines jederzeitigen Austritts, das nicht behindert und auch nicht mit Sanktionen verbunden werden darf..$^{\circ}$ Soweit indes eine Tätigkeit im spezifisch kirchlichen Bereich ausgeübt wird, steht der allgemeine Schutz der Tendenzbetriebe gemäß $\$ I 8 Abs. I Betriebsverfassungsgesetz zur Verfügung. Ein weitergehender Schutz für Kirchen, der im zweiten Absatz dieser Vorschrift vorgesehen ist, ist nicht berechtigt und daher von Ruland zu Recht als verfassungswidrig bezeichnet worden. ${ }^{71}$

\section{Dieter Sterzel \\ Versammlungsfreiheit und Anwesenheitspflicht in der Schule}

\section{Vorbemerkung}

In den letzten Monaten kam es aus unterschiedlichen Anlässen zu öffentlichkeitswirksamen Protestaktionen von Schülern und Schülerinnen, an denen teilweise auch Eltern beteiligt waren. In Emden demonstrierten im November i 988 während der Unterrichtszeit ca. 3000 Schüler und Schülerinnen in der Innenstadt gegen die Verladung von Atommüll im Hafen'. In Wiesmoor/Ostfriesland reagierten Eltern von ro bis I sjährigen Kindern nach dem Absturz zweier Kampfflugzeuge im Januar diesen Jahres in unmittelbarer Nähe einer Schule mit einem zweimaligen Schulboykott gegen militärische Tiefflüge. ${ }^{2}$ In Lüchow-Dannenberg löste die auf Weisung der Schulaufsicht erfolgte Absetzung eines Projekttages zum Thema »Kernenergie

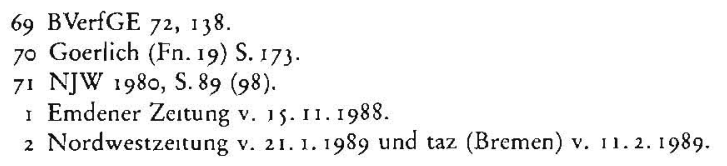

